\title{
Influence of Lymph Node Clinical Target Volume Margin Size on Liver and Kidneys in Three Dimensional Conformal Radiotherapy of Gastric Cancer: A Dosimetric Analysis
}

Hasan Osmic ${ }^{1^{*}}$, Hasukic $\mathbf{S}^{2}$, Hasukic $B^{2}$, Fazlic $\mathbf{S}^{3}$ and Đedovic $E^{3,4}$

${ }^{1}$ Radiotherapy and Oncology Clinic, University Clinical Center Tuzla, Bosnia and Herzegovina

${ }^{2}$ Surgery Clinic, University Clinical Center Tuzla, Bosnia and Herzegovina

${ }^{3}$ Department of Medical Physics and Radiation Protection, University Clinical Center Tuzla, Bosnia and Herzegovina

${ }^{4}$ Department of Physics, University of Tuzla, Bosnia and Herzegovina

"Corresponding author: Hasan Osmic, Radiotherapy and Oncology Clinic, University Clinical Center Tuzla, City of Tuzla, Bosnia and Herzegovina, Tel: +38735303486; Fax number: +38735303389; E-mail: hasan.osmic@ukctuzla.ba

Received date: August 10, 2018; Accepted date: August 31, 2018; Published date: September 07, 2018

Copyright: $\odot 2018$ Osmic $\mathrm{H}$, et al. This is an open-access article distributed under the terms of the Creative Commons Attribution License, which permits unrestricted use, distribution, and reproduction in any medium, provided the original author and source are credited.

\begin{abstract}
The aim of this study was to determine the dosimetrically optimal CTV margin for lymph nodes in patients with gastric cancer treated with 3DCRT. We derived three PTVs for every patient: PTV $5 \mathrm{~mm}$, PTV $7 \mathrm{~mm}$ and PTV 10 $\mathrm{mm}$. For each patient, 3DCRT treatment plans were prepared for each of the three PTVs. From DVHs, for every patient and margin size, mean values and ranges of doses to organs at risk, as well as mean values and ranges of $\mathrm{V}_{28}, \mathrm{~V}_{23}, \mathrm{~V}_{20}$ and $\mathrm{V}_{12}$ for both kidneys were recorded.

Standard deviations of data were also calculated for every particular case. Statistical hypotheses were tested for $\alpha=0.05$ significance level, i.e. differences between the examined groups were considered significant if $p<0.05$.

As a result we got that: The difference is significantly lower in the mean dose to liver for CTV $5 \mathrm{~mm}$ and CTV 10 $\mathrm{mm}$, as well as for CTV $7 \mathrm{~mm}$ and CTV $10 \mathrm{~mm}$; the difference is not significant for CTV $5 \mathrm{~mm}$ and CTV $7 \mathrm{~mm}$; A significantly lower difference is seen in the mean dose to the right and left kidneys for CTV $5 \mathrm{~mm}$ and CTV $10 \mathrm{~mm}$, CTV $7 \mathrm{~mm}$ and CTV $10 \mathrm{~mm}$, while no significant difference is observed for CTV $5 \mathrm{~mm}$ and CTV $7 \mathrm{~mm}$. We concluded that the margin of the nodal CTV of $7 \mathrm{~mm}$ in 3DCRT of gastric cancer dosimetrically spares liver and kidneys better than the CTV $10 \mathrm{~mm}$ margin.
\end{abstract}

Keywords: Gastric cancer; Lymph node CTV; 3DCRT; Organ at risk; Dosimetric analysis; DVH.

Abbreviations: ROG: Radiation Oncology Group; AP: AnteriorPosterior; 3DCRT: Three Dimensional Conformal Radio Therapy; CTV: Clinical Target Volume; PTV: Planning Target Volume; OAR: Organ at Risk; DVH: Dose Volume Histogram; FOV: Field of View; CT: Computed Tomography; MLC: Multileaf Collimator; RLAT: Right Lateral; LLAT: Left Lateral; QUANTEC: Qualitative Analyses of Normal Tissue Effect in the Clinic; ANOVA: Analysis of Variance; EORTC: European Organisation for Rsearch and Treatment of Cancer.

\section{Introduction}

Gastric cancer is the third most common cause of cancer death worldwide. The highest incidence rate is recorded in East Asia, South America and East Europe, whereas the lowest is found in North America [1]. It is a relatively rare type of cancer in the USA with the incidence of 10,000 deaths per year [2]. Surgery is the primary therapy for all operable gastric cancers. Surgery technique depends mostly on cancer localization, size and local status. It should be pointed out that D2 lymphadenectomy is a mandatory standard in such procedures [3].
The role of radiotherapy in gastric cancer treatment has significantly changed in the first decade of the $21^{\text {st }}$ century. It was considered an ineffective method in the treatment of these types of cancers, however, after the introduction of the $3 \mathrm{D}$ planning and other complex radiation techniques and in combination with a sequential and concomitant chemotherapy, it has become more commonly used postoperative method of treatment [4].

Delineation of the tumor CTV is a demanding procedure. It includes CTV of the tumor bed, anastomosis and lymph nodes. When determining the tumor bed and anastomosis, it is recommended to do a preoperative CT $[5,6]$. Stomach lymphatic drainage is very complex and depends on primary localization of gastric cancer. Caravatta et al. have recommended that lymph node stations, depending on the tumor localization, be included in radiotherapy of gastric cancer [7].

3DCRT can be used to create a dosimetric plan where the prescribed dose is drawn near the target volume to increase organs at risk sparing, primarily liver and kidneys. The condition is to precisely contour the target volume in order to avoid under-irradiation and locoregional disease recurrence.

The biggest challenge is to determine the distance between the blood vessels and the largest number of lymph nodes in order to set the optimal CTV of lymph nodes. Pancreas and stomach have very similar vascularization and lymphatic drainage, especially at the level of the 
Citation: Osmic H, Hasukic S, Hasukic B, Fazlic S, Đedovic E (2018) Influence of Lymph Node Clinical Target Volume Margin Size on Liver and Kidneys in Three Dimensional Conformal Radiotherapy of Gastric Cancer: A Dosimetric Analysis. J Nucl Med Radiat Ther 9: 374 . doi: $10.4172 / 2155-9619.1000374$

Page 2 of 6

celiac trunk. However, some authors recommend $10 \mathrm{~mm}$ of CTV margin for pancreatic lymph nodes [8], whereas other authors recommend $5 \mathrm{~mm}$ for lymph nodes in gastroesophageal junction cancer [7].

Lymph nodes are anatomically always located at the same distance from the blood vessels regardless of the type of cancer. It has been shown that $99 \%$ of the lymph nodes in the pelvis are found within a seven millimeter diameter around the blood vessels, and this is due to the fact that those have the same angiogenic origin as the abdominal blood vessels [9].

Since such studies on abdominal blood vessels do not exist, it seems very useful and important to investigate the influence of the size of the lymph nodes CTV margin on the dose received by organs at risk, and to evaluate the safety of the use of conformal radiotherapy for gastric cancer.

The aim of this study was to determine the dosimetrically optimal CTV margin of the lymph nodes so that the selected margin covers all the lymph nodes, and that the doses to organs at risk are as low as possible.

This was done by determining and comparing the influence of the radiation dose to liver and kidneys for the target volumes that included the lymph nodes CTVs of $5 \mathrm{~mm}, 7 \mathrm{~mm}$ and $10 \mathrm{~mm}$, in gastric cancer patients treated with 3DCRT.

\section{Patients and Methods}

\section{Patients}

This factorial analysis study included 40 patients (30 males and 10 females) with operable gastric cancer stage 1B-IV, treated with an adjuvant according to a protocol involving two cycles of XP followed by postoperative chemo-radiotherapy (45 Gy with concurrent capeticabine) followed by two cycle of XP $[10,11]$. Delineation of target volumes was performed on CT images of patients treated at the Department of Oncology, Hematology and Radiotherapy of the University Clinical Center Tuzla. Relevant data on gastric cancer patients were taken from Impac Mosaiq software system for data collection in radiotherapy. 3DCRT plans were prepared for each patient with different nodal volumes: CTV $5 \mathrm{~mm}, 7 \mathrm{~mm}$ and $10 \mathrm{~mm}$.

\section{Simulation and positioning of patients}

Simulation of patients was performed on Philips CT simulator. Patient was in the supine position on a flat table with the hands fixed on the monoaxial holder above the head. Scanning was done according to the standard oncology CT protocol with $5 \mathrm{~mm}$ slice thickness, pitch factor 1, FOV 500, standard resolution and Lung Detail filter. The area from Th10 up to L2/3 was scanned.

\section{Delineation of treatment volumes (CTVs and PTVs) and OARs}

OARs, liver and kidneys, of patients treated with 3DCRT were contoured on CT images. The tumor CTVs were contoured encompassing the tumor bed and anastomosis. After that, 3 CTVs of lymph nodes were contoured for each patient, at $5 \mathrm{~mm}, 7 \mathrm{~mm}$ and 10 $\mathrm{mm}$ around the corresponding blood vessels. At the end, PTVs were defined and included a margin of $15 \mathrm{~mm}$ withinthe tumor and lymph nodes CTVs. In this manner 3 PTVs were obtained for each patient; PTV $5 \mathrm{~mm}$, PTV $7 \mathrm{~mm}$ and PTV $10 \mathrm{~mm}$ (Figure 1).

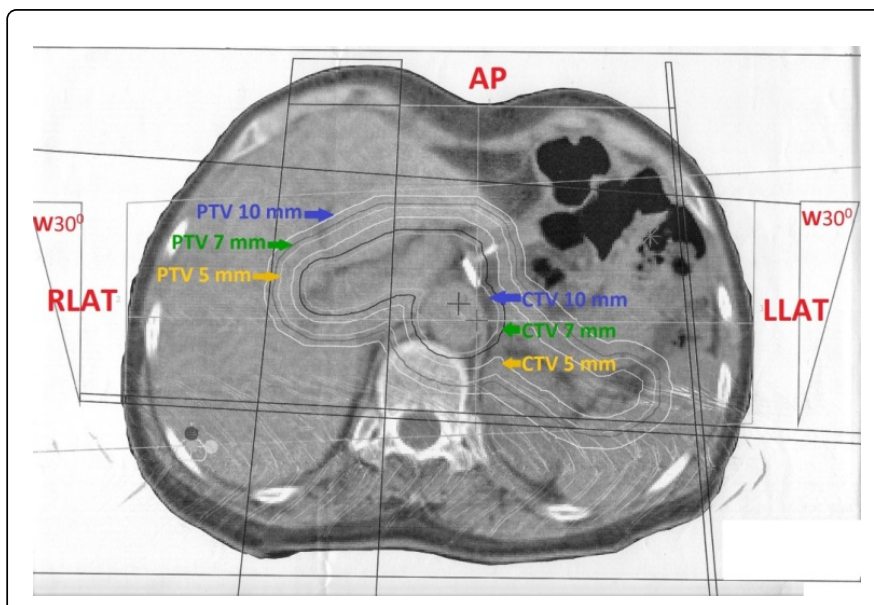

Figure 1: Transversal view of the patient's body with: Contoured CTVs and PTVs, isocentar position and normalization point of the beams, arrangement of AP, RLAT and LLAT beams and wedges for RLAT and LLAT beams.

Using this method, we were able to isolate influence of the lymph nodes CTVs size on the surrounding OARs. Contouring was done in FOCAL radiotherapy planning system. Target volume contouring was done by one single experienced radiation oncologist, all according to the recommendations [7], in order to avoid inter-observer variations in contouring.

\section{Treatment planning and dose calculations}

Treatment plans were made on the Xio treatment planning system. Selection of the treatment fields, gentry angles, beam weights, size of margins of PTV and wedges was done taking into account the specificities of the treatment region:

- Organs at risk are, with one of their parts, captured with the treatment fields, particularly liver.

- The position of organs at risk according to the treatment volume.

- The influence of nonuniform tissue density on the isodose distribution.

- Respiratory motions of the treatment volume and normal structures during the treatment.

For the dose delivery to the treatment volumes (PTV $5 \mathrm{~mm}$, PTV 7 $\mathrm{mm}$ and PTV $10 \mathrm{~mm}$ ), three fields of radiation of $15 \mathrm{MV}$ beam energy were used (Figure 1). The shapes of the fields were adjusted to the treatment volumes using MLC, so that an additional margin of $1 \mathrm{~cm}$ to the PTV was added. In this way, it was ensured that the shapes of the fields remain same for each of PTVs, and the only difference was in their size in respect to the PTV.

The shielding of organs at risk was generated using MLC and it remained the same for each of the three prepared plans. Given the fact that the liver is almost completely in RLAT field and the treatment volume position is more on the left side of the abdomen, the smallest weight of $24.4 \%$ of the prescribed dose in this field was chosen. For the other two fields, AP and LLAT, the same weights of $37.8 \%$ of the 
Citation: $\quad$ Osmic H, Hasukic S, Hasukic B, Fazlic S, Đedovic E (2018) Influence of Lymph Node Clinical Target Volume Margin Size on Liver and Kidneys in Three Dimensional Conformal Radiotherapy of Gastric Cancer: A Dosimetric Analysis. J Nucl Med Radiat Ther 9: 374 . doi: $10.4172 / 2155-9619.1000374$

Page 3 of 6

prescribed dose were chosen. Both lateral fields have the wedge of 30 degrees.

The isocenter of the fields has been set arbitrary taking into account that it is as close as possible to the center of the treatment volume. The normalization point for three fields is very close to the isocenter. For dosimetric analysis to be meaningful, it was ensured that at least $95 \%$ of PTV was covered with $95 \%$ of the prescribed dose. The superposition algorithm was used to calculate the isodose distribution.

\section{Treatment plan evaluation}

After the treatment plans have been completed, their evaluation using DVH was performed. For the purpose of the dosimetric analysis, the mean dose values to organs at risk, as well as the values of $\mathrm{V}_{28}, \mathrm{~V}_{23}$, $\mathrm{V}_{20}$ and $\mathrm{V}_{12}$ for right and left kidneys were read from DVHs. Parameters for DVHs for liver and kidneys were also analyzed according to the QUANTEC recommendations [12].

\section{Statistical data analysis}

Standard methods of descriptive statistics (mean with standard deviation and range from minimum to maximum) were used in statistical data analysis. Statistical significance of the differences between the target groups was evaluated using ANOVA test at the significance level of $\alpha=0.05$, i.e. differences between the target groups were considered significant if $\mathrm{p}<0.05$. Data were analyzed using Arcus Quickstat statistical software (version 1.0.0.8.8, Medical Computing).

\section{Results}

The study included 40 patients, 30 male and 10 female patients (ratio of men to women was 3:1). The average age of patients was 59, ranging from 37 to 78 years old. Further analysis showed that gastric cancer was the highest in the 50 to 60 age group and 60 to 70 years. The lowest number of gastric cancers was noted in the age group 30 to 40 years. With respect to tumor size, 18 patients had a tumor size of 2 to $5 \mathrm{~cm}$.

All 40 patients had an adenocarcinoma by histological type. According to localization, the largest number of tumors were found in antrum [12], then corpus, pylorus, while the smallest number was in cardia, in 5 patients. Thirteen patients had up to 6 metastasis in the lymph nodes, and two patients had metastasis in more than 15 lymph nodes, while twenty-five patients had positive lymph nodes. Eighteen patients had a T4 tumor stage, while the T1 stage was the least present among patients. Twenty-nine patients had G3 (poorly differentiated tumor), eighteen patients had G2 (moderately differentiated tumor) and two patients had G1 (well differentiated tumor) (Table 1).

\begin{tabular}{|l|l|l|}
\hline Variable & Subgroup & Number \\
\hline \multirow{4}{*}{ T stage } & T1 & 1 \\
\cline { 2 - 3 } & T2 & 9 \\
\cline { 2 - 3 } & T3 & 12 \\
\hline \multirow{4}{*}{ Tumour localization } & T4 & 18 \\
\hline & Cardia & 5 \\
\cline { 2 - 3 } & Corpus & 14 \\
\cline { 2 - 3 } & Antrum & 16 \\
\hline
\end{tabular}

\begin{tabular}{|l|l|l|}
\hline \multirow{2}{*}{ N stage } & Pylorus & 5 \\
\hline & N1 & 13 \\
\cline { 2 - 3 } & N2 & 20 \\
\cline { 2 - 3 } & N3 & 7 \\
\hline \multirow{3}{*}{ Pathohistological gradus } & I & 3 \\
\cline { 2 - 3 } & II & 18 \\
\cline { 2 - 3 } & III & 29 \\
\hline
\end{tabular}

Table 1: Pathohistological characteristics.

Table 2 shows DVHs results for liver and both kidneys in 3DCRT plans made for target volumes including the lymphnodes CTVs of 5 $\mathrm{mm}, 7 \mathrm{~mm}$ and $10 \mathrm{~mm}$. It also shows the mean with standard deviation, as well as the minimum and maximum range in grays (Gy).

The mean dose value delivered to the liver at $10 \mathrm{~mm}$ CTV is beyond the constraint for liver, whereas doses of $5 \mathrm{~mm}$ and $7 \mathrm{~mm}$ CTVs were within the allowed limits. The average mean dose to the right kidney at $10 \mathrm{~mm} \mathrm{CTV}$ is at the constraint borderline, while doses of $5 \mathrm{~mm}$ and 7 $\mathrm{mm}$ CTVs are within the allowed limits.

The average dose to the left kidney is the best at CTV $5 \mathrm{~mm}$, whereas at CTV $7 \mathrm{~mm}$ is $1.34 \mathrm{~Gy}$ above the constraint. The average mean dose to the left kidney at CTV $10 \mathrm{~mm}$ is 22.16 Gy which is far beyond the constraint.

\begin{tabular}{|c|c|c|c|c|}
\hline OAR & CTV & Mean dose [Gy] & $\begin{array}{l}\text { SD } \\
{[G y]}\end{array}$ & Min.-Max. dose [Gy] \\
\hline \multirow{3}{*}{ Liver } & CTV 5 mm & 30.05 & 2.28 & $24.25-35.15$ \\
\hline & CTV $7 \mathrm{~mm}$ & 31.17 & 2.29 & $25.20-36.22$ \\
\hline & $\begin{array}{l}\text { CTV } \\
\mathrm{mm}\end{array}$ & 33.07 & 2.18 & $27.63-37.82$ \\
\hline \multirow{3}{*}{$\begin{array}{l}\text { Right } \\
\text { kidney }\end{array}$} & CTV $5 \mathrm{~mm}$ & 13.84 & 4.35 & $4.81-26.39$ \\
\hline & CTV $7 \mathrm{~mm}$ & 15.29 & 4.33 & $5.19-27.98$ \\
\hline & $\begin{array}{ll}\text { CTV } & 10 \\
\mathrm{~mm} & \end{array}$ & 18.08 & 4.56 & $9.05-31.26$ \\
\hline \multirow{3}{*}{ Left kidney } & CTV $5 \mathrm{~mm}$ & 17.95 & 5.54 & $5.93-31.50$ \\
\hline & CTV $7 \mathrm{~mm}$ & 19.34 & 5.81 & $6.26-32.29$ \\
\hline & $\begin{array}{l}\text { CTV } \\
\mathrm{mm}\end{array}$ & 22.16 & 5.96 & $6.46-34.61$ \\
\hline
\end{tabular}

Table 2: Mean doses, SDs and range values for OARs in the case of different CTV margin sizes.

Table 3 shows the DVH results of a mean percentage of organ volume receiving doses of $28 \mathrm{~Gy}, 23 \mathrm{~Gy}, 20 \mathrm{~Gy}$ and $12 \mathrm{~Gy}$ for the right and left kidneys. From the presented results, it is evident that, according to QUANTEC, in the case of CTV of $5 \mathrm{~mm}$ values of $\mathrm{V}_{28}$, $\mathrm{V}_{23}, \mathrm{~V}_{20}$ and $\mathrm{V}_{12}$ for the right kidney, and only the value $\mathrm{V} 12$ for the left kidney are within the allowed limits. Similar results are shown in the case of CTV of $7 \mathrm{~mm}$ for the right kidney, but all Vs values are beyond the constraints for the left kidney. In the case of CTV of $10 \mathrm{~mm}$ all Vs values for both kidneys are beyond the constraints. 
Citation: Osmic H, Hasukic S, Hasukic B, Fazlic S, Đedovic E (2018) Influence of Lymph Node Clinical Target Volume Margin Size on Liver and Kidneys in Three Dimensional Conformal Radiotherapy of Gastric Cancer: A Dosimetric Analysis. J Nucl Med Radiat Ther 9: 374 . doi: $10.4172 / 2155-9619.1000374$

Page 4 of 6

\begin{tabular}{|c|c|c|c|c|c|c|c|c|c|c|c|c|c|}
\hline \multirow{2}{*}{ OAR } & \multirow{2}{*}{ CTV } & \multicolumn{4}{|c|}{$V x[\%]^{*}$} & \multicolumn{4}{|c|}{ SD [\%] } & \multicolumn{4}{|c|}{ Min.-Max. volume [\%] } \\
\hline & & $\mathbf{v}_{28}$ & $\mathbf{v}_{23}$ & $\mathbf{v}_{20}$ & $\mathbf{v}_{12}$ & $\mathbf{v}_{28}$ & $\mathbf{v}_{23}$ & $\mathbf{v}_{20}$ & $\mathbf{v}_{12}$ & $\mathbf{v}_{28}$ & $\mathbf{v}_{23}$ & $\mathbf{v}_{20}$ & $\mathbf{v}_{12}$ \\
\hline \multirow{6}{*}{$\begin{array}{l}\text { Right } \\
\text { Kidney }\end{array}$} & \multirow{2}{*}{$\begin{array}{l}\text { CTV } 5 \\
\mathrm{~mm}\end{array}$} & \multirow{2}{*}{18.6} & \multirow{2}{*}{23.9} & \multirow{2}{*}{27.4} & \multirow{2}{*}{43.3} & \multirow{2}{*}{11.4} & \multirow{2}{*}{12.5} & \multirow{2}{*}{13.2} & \multirow{2}{*}{14.4} & $1.5-$ & $13.2-$ & 4.6- & $11.0-$ \\
\hline & & & & & & & & & & 55.1 & 61.6 & 65.6 & 79.5 \\
\hline & \multirow{2}{*}{$\begin{array}{l}\text { CTV } \\
\mathrm{mm}\end{array}$} & \multirow{2}{*}{22.4} & \multirow{2}{*}{27.9} & \multirow{2}{*}{31.6} & \multirow{2}{*}{47.7} & \multirow{2}{*}{11.8} & \multirow{2}{*}{12.9} & \multirow{2}{*}{13.4} & \multirow{2}{*}{13.9} & $6.6-$ & 4.3- & $6-$ & $15.0-$ \\
\hline & & & & & & & & & & 72.3 & 66.7 & 70.5 & 82.7 \\
\hline & \multirow{2}{*}{$\begin{array}{l}\text { CTV } 10 \\
\mathrm{~mm}\end{array}$} & \multirow{2}{*}{34.1} & \multirow{2}{*}{35.1} & \multirow{2}{*}{39.3} & \multirow{2}{*}{56.4} & \multirow{2}{*}{11.8} & \multirow{2}{*}{13.8} & \multirow{2}{*}{14} & \multirow{2}{*}{14.4} & $6.4-$ & $10.8-$ & 14.2- & 26.4- \\
\hline & & & & & & & & & & 22.3 & 75.7 & 78.9 & 89.8 \\
\hline \multirow{6}{*}{ Left Kidney } & \multirow{2}{*}{$\begin{array}{l}\text { CTV } 5 \\
\mathrm{~mm}\end{array}$} & 297 & 347 & 30 & 511 & 140 & 151 & 150 & 155 & $4.6-$ & $6.9-$ & 8.9- & $22.9-$ \\
\hline & & & & & & & & & & 70.1 & 76.4 & 79.6 & 88.4 \\
\hline & CTV 7 & 231 & 304 & 110 & {$[0$,} & (4) & 45 & 10 & I & 6.6- & $9.2-$ & 6- & $25.4-$ \\
\hline & $\mathrm{mm}$ & & & & & & & & 10.5 & 72.3 & 78.4 & 70.5 & 90.2 \\
\hline & CTV 10 & 403 & 158 & 193 & 661 & 155 & 164 & 14 & 153 & 10.1- & $13.4-$ & $11.4-$ & 30.3- \\
\hline & $\mathrm{mm}$ & 40.0 & 40.0 & 4.5 & 00.1 & 10.5 & 10.4 & 14 & 10.0 & 80.9 & 85.4 & 81.7 & 93.8 \\
\hline
\end{tabular}

Table 3: Mean percentage of organ volume that received x Gy dose for different CTVs.

The difference in the DVH parameters for the defined target volumes for the liver and kidneys is shown in Table 4 . The difference is significantly lower in the mean dose to liver for volumes CTV $5 \mathrm{~mm}$ and CTV $10 \mathrm{~mm}$, as well as for CTV $7 \mathrm{~mm}$ and CTV $10 \mathrm{~mm}$. On the other hand, the difference is not significant for volumes CTV $5 \mathrm{~mm}$ and CTV $7 \mathrm{~mm}$.

Also, significantly lower difference is seen in the average mean dose to the right kidney for volumes CTV $5 \mathrm{~mm}$ and CTV $10 \mathrm{~mm}$, CTV 7 $\mathrm{mm}$ and CTV $10 \mathrm{~mm}$, while no significant difference is observed for CTV $5 \mathrm{~mm}$ and CTV $7 \mathrm{~mm}$. The difference in the average mean dose to the left kidney for volumes CTV $7 \mathrm{~mm}$ and CTV $10 \mathrm{~mm}$ is on the CTV $7 \mathrm{~mm}$ side ( $\mathrm{p}=0.07$ ), whereas the difference is significant for CTV $5 \mathrm{~mm}$ and CTV $10 \mathrm{~mm}$. There was no significant difference for volumes CTV $5 \mathrm{~mm}$ and CTV $7 \mathrm{~mm}$.

\begin{tabular}{|c|c|c|c|}
\hline OAR & CTV & Difference in mean dose (Cl 95\%) [Gy] & p-value \\
\hline \multirow{3}{*}{ Liver } & CTVs $5 \mathrm{~mm}$ vs. $10 \mathrm{~mm}$ & $-3.0(-4.2$ to -1.8$)$ & $<0.0001$ \\
\hline & CTVs $7 \mathrm{~mm}$ vs. $10 \mathrm{~mm}$ & $-1.9(-3.1$ to -0.7$)$ & 0.0007 \\
\hline & CTVs $5 \mathrm{~mm}$ vs. $7 \mathrm{~mm}$ & $-1.1(-2.3$ to 0.8$)$ & 0.0740 \\
\hline \multirow{3}{*}{ Right Kidney } & CTVs $5 \mathrm{~mm}$ vs. $10 \mathrm{~mm}$ & $-4.2(-6.6$ to -1.9$)$ & 0.0001 \\
\hline & CTVs $7 \mathrm{~mm}$ vs. $10 \mathrm{~mm}$ & $-2.8(-5.1$ to -0.4$)$ & 0.0010 \\
\hline & CTVs $5 \mathrm{~mm}$ vs. $7 \mathrm{~mm}$ & $-1.5(-3.8$ to 0.9$)$ & 0.3000 \\
\hline \multirow{3}{*}{ Left Kidney } & CTVs $5 \mathrm{~mm}$ vs. $10 \mathrm{~mm}$ & $-4.2(-7.3$ to -1.1$)$ & 0.0040 \\
\hline & CTVs $7 \mathrm{~mm}$ vs. $10 \mathrm{~mm}$ & $-2.8(-5.9$ to 0.2$)$ & 0.0700 \\
\hline & CTVs $5 \mathrm{~mm}$ vs. $7 \mathrm{~mm}$ & $-1.4(-4.4$ to 1.7$)$ & 0.5300 \\
\hline
\end{tabular}

Table 4: DVH parameters difference for liver and kidneys in tested target volumes.

\section{Discussion and Conclusion}

This is the first study to investigate the issue of elective lymph node clinical target volume margin in gastric cancer from the dosimetric aspect, because precise anatomic distance between the lymph nodes and the corresponding blood vessels can only be demonstrated by an anatomic study. No similar studies have been published on this subject yet, but there are a large number of studies that have demonstrated that lymph node dissection in gastric cancer is important because it leads to a higher survival rate and can serve as a prognostic parameter in treatment of gastric cancer [13-15]. 
Citation: Osmic H, Hasukic S, Hasukic B, Fazlic S, Đedovic E (2018) Influence of Lymph Node Clinical Target Volume Margin Size on Liver and Kidneys in Three Dimensional Conformal Radiotherapy of Gastric Cancer: A Dosimetric Analysis. J Nucl Med Radiat Ther 9: 374 . doi: $10.4172 / 2155-9619.1000374$

Page 5 of 6

Determining the clinical target volume for the lymph nodes can be very demanding because one must determine the lymph node regions and the distance around the corresponding blood vessels within which the majority lymph nodes are present. Even when the regions are set and determined, the distance estimation is quite complex. With a larger margin the target will not be missed, but the dose to OAR might be higher than in the case of a smaller margin. Lymph nodes can be missed with a smaller margin, but OAR will be spared.

The EORTC-ROG expert group recommends that the margin around the corresponding blood vessels be $5 \mathrm{~mm}$ [7]. Pancreatic cancer experts claim that the margin around the same corresponding blood vessels that also belong to the stomach should be $10 \mathrm{~mm}$ [8], but it has been shown that $99 \%$ of the lymph nodes in the pelvis are located at $7 \mathrm{~mm}$ distance from the blood vessels [9]. Furthermore, interobserver variations in delineation of CTV in gastric cancer radiotherapy are statistically significant [16].

The results of this study showed that the average mean dose to liver for CTV $10 \mathrm{~mm}$ is $33.0 \mathrm{~Gy}$, CTV $5 \mathrm{~mm} 30.0 \mathrm{~Gy}$ and CTV $7 \mathrm{~mm} 31.2$ Gy. In the study by El-Hossiny et al. [17], the average mean dose to liver was 20.0 Gy, in Leong et al. [18] it was 22.0 Gy and in Soyfer et al. [19] 24.0 Gy. Due to the fact that the arrangement of conformal fields in the above-mentioned studies was very similar, it is evident that the difference in CTV defining is caused by the differences in average mean liver doses. The difference in the average mean liver doses is also significantly lower for CTV $5 \mathrm{~mm}$ and CTV $10 \mathrm{~mm}$, CTV $7 \mathrm{~mm}$ and CTV $10 \mathrm{~mm}$, and there are no significant differences for CTV $5 \mathrm{~mm}$ and CTV $7 \mathrm{~mm}$. The $7 \mathrm{~mm}$ margin is equally safe as $5 \mathrm{~mm}$ margin when it comes to liver dose, but in the former case we can ensure that more lymph nodes are covered.

The right kidney average mean dose for CTV $10 \mathrm{~mm}$ is at the constraint borderline (18.1 Gy), doses for CTV $5 \mathrm{~mm}$ and CTV $7 \mathrm{~mm}$ are within the allowed limits. The left kidney average mean dose is the best for CTV $5 \mathrm{~mm}$ while CTV $7 \mathrm{~mm}$ is 1.3 Gy above the allowed limit. The left kidney average mean dose for CTV $10 \mathrm{~mm}$ is $22.2 \mathrm{~Gy}$ which is far beyond the constraint.

There is a significantly lower difference in the average mean dose to the right kidney for CTV $5 \mathrm{~mm}$ and CTV $10 \mathrm{~mm}$, CTV $7 \mathrm{~mm}$ and CTV $10 \mathrm{~mm}$, while there is no difference for CTV $5 \mathrm{~mm}$ and CTV 7 $\mathrm{mm}$. The difference in the average mean dose to the left kidney for CTV $7 \mathrm{~mm}$ and $10 \mathrm{~mm}$ is on the CTV $7 \mathrm{~mm}$ side ( $\mathrm{p}=0.07$ ). On the other hand, there is a significant difference for CTV $5 \mathrm{~mm}$ and CTV 10 $\mathrm{mm}$. There is no significant difference for CTV $5 \mathrm{~mm}$ and CTV $7 \mathrm{~mm}$. If we analyze the volumes of the right and the left kidney that have received doses of $12.0 \mathrm{~Gy}, 20.0 \mathrm{~Gy}, 23.0 \mathrm{~Gy}$ and $28.0 \mathrm{~Gy}$, we can see that the nodal margin at CTV $7 \mathrm{~mm}$ provides dosimetric safety not only when it comes to the mean dose to the kidneys volume (Tables 2-4). In the study by El-Hossiny et al. [17] the average mean dose to the right kidney was $13.0 \mathrm{~Gy}$ and $17.0 \mathrm{~Gy}$ to the left kidney; $\mathrm{V}_{20}$ was $30 \%$ for the right kidney and $34 \%$ for the left kidney, corresponding to the results of this study and for $7 \mathrm{~mm} \mathrm{CTV}$.

In conclusion, the margin of the nodal CTV of $7 \mathrm{~mm}$ in 3DCRT of gastric cancer dosimetrically spares the liver and kidneys better than the $10 \mathrm{~mm}$ margin. There is no difference between $7 \mathrm{~mm}$ and $5 \mathrm{~mm}$ margins, but $7 \mathrm{~mm}$ is expected to provide a better lymphatic coverage.

\section{Ethical Approval}

Received from the department for Scientific research and professional development of the University Clinical Centre Tuzla (04-09/2-40/16 date: 15.06.2016.). Study was conducted in accordance to the principles of the Declaration of Helsinki.

\section{Declaration of Authorship}

$\mathrm{HO}, \mathrm{GM}$ and SF designed the study. $\mathrm{HO}, \mathrm{EĐ}$ and SF performed data acquisition. $\mathrm{HO}, \mathrm{SH}, \mathrm{E}, \mathrm{GM}, \mathrm{SF}$ and $\mathrm{BH}$ analyzed and interpreted the data. $\mathrm{HO}$ and $\mathrm{SH}$ drafted the manuscript. $\mathrm{HO}$ is responsible for all aspects of the work, ensuring the accuracy or integrity of any part of the work.

All co-authors critically reviewed the manuscript and gave their final approval of the version of the manuscript to be published.

\section{Conflict of Interest}

The authors declare that they have no conflict of interest. This study was not sponsored by any external organisation.

\section{References}

1. World Cancer Report (2014) World Health Organization. ISBN 9283204298.

2. Bethesda (2014) National Cancer Institute: $\mathrm{PDQ}^{\circ}$ Stomach (Gastric) Cancer Screening. http://www. 2015.cancer.gov/cancertopics/pdq/ screening/gastric/HealthProfessional.

3. Brancato S, Miner TJ (2008) Surgical management of gastric cancer: Review and consideration for total care of the gastric cancer patient. Curr Treat Options Gastroenterol 11:109-118.

4. SA McCloskey, GY Yang (2009) Benefits and challenges of radiation therapy in gastric cancer: techniques for improving outcomes. Gastrointest Cancer Res 3: 15-19.

5. Soyfer V, Corn BW, Melamud A (2007) Three-dimensional non-coplanar conformal radiotherapy yields better results than traditional beam arrangements for adjuvant treatment of gastric cancer. Int J Radiat Oncol Biol Phys 69: 364-369.

6. Morganti AG, Di Castelnuovo A, Massaccesi M (2013) Planning comparison between standard and conformal 3D techniques in postoperative radiotherapy of gastric cancer: A systematic review. Br J Radiol 86: 20130274.

7. Matzinger O, Gerber E, Bernstein Z (2009) EORTC-ROG expert opinion: Radiotherapy volume and treatment guidelines for neoadjuvant radiation of adenocarcinomas of the gastroesophageal junction and the stomach. Radiother Oncol 92:164-175.

8. Caravatta L, Sallustio G, Pacelli F (2012) Clinical target volume delineation including elective nodal irradiation inpreoperative and definitive radiotherapy of pancreatic cancer. Radiat Oncol 7: 86.

9. Lim K, Small W Jr, Portelance L, Creutzberg C (2011) Consensus guidelines for delineation of clinical target volumefor intensitymodulated pelvic radiotherapy for the definitive treatment ofcervix cancer. Int J Radiat Oncol Biol Phys 79: 348-355.

10. Jin Z, Jiang W, Wang L (2015) Biomarkers for gastric cancer: Progression in early diagnosis and prognosis (Review). Oncol Lett 9: 1502-1508.

11. Lee J, Lim DH, Kim S, Park SH, Park Jo, et al. (2012) Phase III trial comparing capecitabine plus cisplatin versus capecitabine plus cisplatin with concurrent capecitabine radiotherapy in completely resected gastric cancer with D2 lymph node dissection: The ARTIST trial. J Clin Oncol 30:268-273.

12. Marks LB, Yorke DE, Jackson A (2010) Use of normal tissue complication probability models in the clinic. Int J Radiat Oncol Biol Phys 76: S10-S19. 
Citation: $\quad$ Osmic H, Hasukic S, Hasukic B, Fazlic S, Đedovic E (2018) Influence of Lymph Node Clinical Target Volume Margin Size on Liver and Kidneys in Three Dimensional Conformal Radiotherapy of Gastric Cancer: A Dosimetric Analysis. J Nucl Med Radiat Ther 9: 374 . doi: $10.4172 / 2155-9619.1000374$

Page 6 of 6

13. Liu J, Su M, Wang J (2016) A novel grade-lymph node ratio mode predicts the prognosis of the advanced gastric cancer patients after neoadjuvant radiotherapy. Oncotarget 8: 14058-14067.

14. Chen QY, Huang CM, Zheng CH (2016) Do preoperative enlarged lymph nodes affect the oncologic outcome of laparoscopic radical gastrectomy for gastric cancer? Oncotarget 8: 8825-8834.

15. Lee JH, Kang JW, Nam BH (2016) Correlation between lymph node count and survival and areappraisal of lymph node ratio as a predictor of survival in gastric cancer: A multi-institutional cohort study. Eur J Surg Oncol 43: 432-439.

16. Li GC, Zhang Z, Ma XJ (2016) Variations in CT determination of target volume with active breath co-ordinate in radiotherapy for post-operative gastric cancer. Br J Radiol 89:20150332.
17. El-Hossiny HA, Diab NA, El-Taher MM (2009) A comparative dosimetric study of adjuvant $3 \mathrm{D}$ conformal radiotherapy for operable stomach cancer versus AP-PA conventional radiotherapy in NCI-Cairo. J Egypt Natl Canc Inst 21: 197-202.

18. Leong T, Willis D, Joon DL, Condron S, Hui A (2005) 3D conformal radiotherapy for gastric cancer-results of a comparative planning study. Radiother Oncol 74: 301-306.

19. Soyfer V, Corn BW, Melamud A (2007) Three-dimensional non-coplanar conformal radiotherapy yields better results than traditional beam arrangements for adjuvant treatment of gastric cancer. Int J Radiat Oncol Biol Phys 69: 364-369. 